\title{
Bioefficacy of Flindersine against Helicoverpa armigera Hübner, Spodoptera litura Fabricius, Anopheles stephensis Liston. and Culex quinquefasciatus Say.
}

\author{
Veeramuthu Duraipandiyan ${ }^{1,2^{*}}$, Kathirvelu Baskar ${ }^{2,3}$, Chelliah Muthu ${ }^{2}$, Savarimuthu \\ Ignacimuthu $^{1,2,4}$ and Naif Abdullah Al-Dhabi ${ }^{1}$ \\ ${ }^{I}$ Department of Botany and Microbiology; Addiriyah Chair for Environmental studies; College of Science; King \\ Saud University; Riyadh - Kingdom of Saudi Arabia. ${ }^{2}$ Entomology Research Institute; Loyola College; Chennai - \\ India. ${ }^{3}$ Bioscience Research Foundation; Porur; Chennai - India. ${ }^{4}$ Visiting Professor Program, Deanship of \\ Scientific Research; College of Science; King Saud University;Riyadh - Saudi Arabia
}

\begin{abstract}
Flindersine, an alkaloid isolated from Toddalia asiatica, was evaluated for their antifeedant, larvicidal and growth inhibitory activities against Helicoverpa armigera, Spodoptera litura and larvicidal activity against vector mosquitoes Anopheles stephensi and Culex quinquefasciatus. For this, leaf disc no choice method was used for agricultural pests; larvicidal activity was tested on second and fourth instar larvae for mosquitoes at different concentrations. Flindersine showed antifeedant, larvicidal and growth inhibitory activities against $\mathrm{H}$. armigera and S. litura and larvicidal activity against vector mosquitoes An. stephensi and Cx. quinquefasciatus. It showed high regression $\left(R^{2}\right)$ values of 0.91 and 0.87 against $\mathrm{H}$. armigera and $\mathrm{S}$. litura, respectively for antifeedant activity. Flindersine exhibited more than $65 \%$ larvicidal activity against both the pests with LC $C_{50}$ values of 443.04 and 568.88 ppm and $R^{2}$ values of 0.87 and 0.90 against $\mathrm{H}$. armigera and $\mathrm{S}$. litura, respectively. The larval and pupal duration of tested insects increased to more than five days at 1000 ppm when compared with the control. The adult emergence was reduced when the concentration of flindersine was increased. At 1000 ppm, no adult emergence was observed in both the pests. Flindersine exhibited 100\% larvicidal activity against both the tested mosquitoes at 20 ppm concentration, which showed $L C_{50}$ values of $2.90,4.19,1.68$ and 2.71 ppm for $2^{\text {nd }}$ and $4^{\text {th }}$ instar larvae of Cx. quinquefasciatus and An. Stephensi, respectively. High regression values were observed for antifeedant, larvicidal and growth inhibitory activities. Flindersine could be used to develop an ecofriendly pesticide formulation to control the agricultural pests and vector mosquitoes.
\end{abstract}

Key words: Flindersine, Adult emergence, Antifeedant, Growth inhibitiory, Larvicidal

\section{INTRODUCTION}

Chemical pesticides have been playing a major role in plant protection to increase the production. At the same time, they also cause severe adverse health effects on humans and animals. Indiscriminate use of synthetic pesticides in agricultural ecosystem causes environmental pollution, development of insect resistance and more importantly affects the non-target organisms; they also reduce the natural enemy populations (Youn et al. 2003). Hence, there is a need for ecofriendly pesticides. Bioesticides are considered an alternatives to chemical pesticides; they are ecofriendly and also possess many modes of action. Several studies have been made on

*Author for correspondence: avdpandiyan@yahoo.co.in 
plant-based pesticides. According to Balick et al. (1995) less than $1 \%$ of bioactive natural compounds have been chemically identified from plant source.

Many plant extracts, semi purified and purified compounds have been studied for insect control properties by many researchers all over the world. Secondary metabolites from plants play a key defensive role against the insects and act as antifeedants, oviposition deterrents and growth inhibitors (Isman 2002). Antifeedants are important factors in insect-plant interaction and are useful to study the field crop pests' behavior (Usher et al. 1988). Natural compounds such as Juglone, plumbagin and emodin showed antifeedancy against Trichoplusia ni (Hübner) (Akhtar et al. 2012). Plant derived compounds are toxic to insect pests; some of the compounds are highly active (Salehzadeh et al. 2002). Munoz et al. (2013) reported that Calceolaria talcana (Calceolariaceae) derived extracts revealed antifeedant, larvicidal, and growth inhibitory activities (reduced the adult emergence, pupal weight and pupation) against $S$. frugiperda (J.E. Smith). Ar-turmerone from Curcuma longa (Zingiberaceae) inhibited the growth (width of head capsule, length and weight) of $S$. frugiperda (Tavares et al. 2013). Rhodojaponin-III, a grayanoid diterpene from Rhododendron molle (Ericaceae) acted as antifeedant, stomach poison, contact toxicant and insect growth inhibitor against Pieris rapae Linnaeus (Zhong et al. 2006). Toddalia asiatica from Rutaceae family has many medicinal properties. It is widely used as a folk medicine in India. The Kani tribes use the extract of this plant to cure skin diseases (Ayyanar and Ignacimuthu 2005). The extracts of this plant exhibited strong insecticidal properties against Aedes aegypti Linnaeus, A. albopictus Skuse and Culex quinquefasciatus Say (Borah et al. 2010; Liu et al. 2013; Nyahanga et al. 2013); and larvicidal and antifeedant properties against Helicoverpa armigera Hübner (Sundararajan and Kumuthakalavalli 2001; Ramya et al. 2009). H. armigera is a polyphagous pest causing serious damage to many agricultural and horticultural crops such as cotton, tomato, brinjal, chickpea, pigeonpea, chilli, maize, and sorghum (Manjunath et al. 1989; Talekar et al. 2006). Over 112 plant species from 44 families of plants are infested by this insect. In India, this is reported to feed on over 40 species (Ramana et al. 1988; Dhir et al. 1992). Larvae feed on the leaves, flowers and even pods (Kriti et al. 2014). Spodoptera litura Fabricius is a polyphagous pest. It also attacks more than 100 crops (Talukder and Howse 1994). Both $H$. armigera and S. litura have developed resistance against synthetic pesticides (Kumar and Reghupathy 2001; Ramasubramaniam and Regupathy 2004).

Mosquitoes are responsible for spreading diseases like malaria, Japanese encephalitis, yellow fever, dengue and filariasis. Among the diseases, $C x$. quinquefasciatus spread the lymphatic filariasis which is more common in Indian subcontinent (Rajasekariah et al. 1991). Malaria is a deadly disease in India, which is spread by An. stephensi. Large numbers of programmes are conducted for mosquito larvicidal controls at the breeding sites, which are most common method in India to control and manage the mosquitoes. Fogging is the temporary method to control the adult mosquitoes; the most efficient method is to manage the mosquito population by killing the larvae. Many researchers highlighted the effects of botanical pesticides on vector mosquitoes. Senthilkumar et al (2008) found that essential oil from Blumea mollis (Asteraceae) exhibited larvicidal activity on $C x$. quinquefasciatus. Herbal formulation Ponneem exhibited larvicidal activity against Aedes aegypti Linnaeus and A. albopictus (Maheswaran and Ignacimuthu 2012). Benzene and their fractions of Citrullus vulgaris Cucurbitaceae exhibited larvicidal activity against Anopheles stephensi Liston and A. aegypti (Mullai et al. 2008). The present study was evaluated the antifeedant and toxic effects of flindersine isolated from T. asiatica against $H$. armigera, S. litura, An. Stephensi and Cx. quinquefasciatus.

\section{MATERIAL AND METHODS}

\section{Plant material}

The leaves of Toddalia asiatica Lam. (Rutaceae) were collected from the forest area of Kanchipuram District, Tamil Nadu, India. The plant was identified by a taxonomist at the Department of Botany, Madras Christian College, Chennai, India. The voucher specimen (ERIC-D78) was deposited at the Entomology Research Institute, Loyola College, Chennai, India.

\section{Flindersine}

The isolation and identification of flindersine (Fig. 1) was done following Duraipandiyan and Ignacimuthu (2009). 


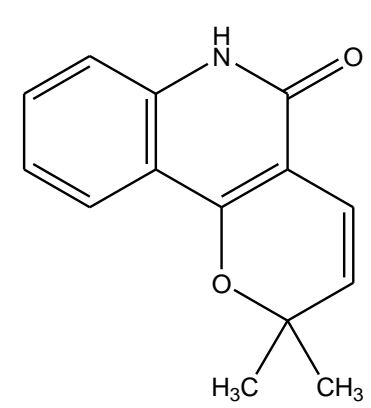

Figure 1 - Flindersine (2,6-dihydro-2,2-dimethyl$5 \mathrm{H}$-pyrano [3,2-c] quinoline-5-one$9 \mathrm{cl})$ isolated from Toddalia asiatica leaves.

\section{Insect culture}

Larvae of $H$. armigera and S. litura were collected from the farmers' field in Monnavedu, Thirvallur district of Tamil Nadu. H. armigera larvae were reared individually in a plastic container and fed regularly with bhendi fruit (Abelmoschus esculentus L.) (Malvaceae); S. litura larvae were offered castor leaves. The larvae were kept till they became pupae under the laboratory conditions $\left(27 \pm 2^{\circ} \mathrm{C}\right.$ and $75 \pm 5 \%$ relative humidity). Sterilized soil was provided for pupation. After pupation, the pupae were collected from the soil and placed inside the cage for the emergence of adults. Cotton soaked with $10 \%$ honey solution mixed with a few drops of multivitamins was provided for adult feeding to increase the fecundity. Potted cowpea plants were kept for $H$. armigera, and groundnut plants were provided for $S$. litura separately inside the adult emergence cages for egg laying. After hatching, the larvae were collected from the respective cages and fed with standard artificial diet as recommended by Koul et al. (1997) for $H$. armigera and castor leaf for S. litura.

\section{Rearing of vector mosquitoes}

Larvae of An. stephensi and $C x$. quinquefasciatus were derived from various places with stagnant water bodies in Chennai, India. They were cultured in the laboratory for many generations and were maintained continuously at laboratory conditions $\left(27 \pm 2^{\circ} \mathrm{C}, \quad 75-85 \% \quad \mathrm{RH}\right.$ and photoperiod of 14:10h, light/dark). Full development from the egg to adult lasted for about 3-4 weeks. Larvae were fed on finely ground dog biscuit mixed with yeast extract in the ratio of 3:1. The water was changed daily to avoid scum formation. Pupae were transferred from the trays to a plastic cup containing tap water and placed inside the cage $(30 \times 30 \times 30 \mathrm{~cm}$ dimension) for adult emergence. The emerged adults were reared in respective cages. The adult colony was provided with ten percent sucrose solution and it was periodically blood-fed on restrained rats. After three days, ovitrap was kept in the cages for egg laying and the eggs were collected and transferred to enamel trays. Newly hatched second and fourth instar larvae were used for the present experiments.

\section{Antifeedant activity}

Antifeedant activity of flindersine was studied using leaf disc no-choice method. Fresh cotton $(H$. armigera) and castor (S. litura) leaf discs of $4 \mathrm{~cm}$ diameter were punched using a cork borer. They were dipped in $125,250,500$ and $1000 \mathrm{ppm}$ flindersine and azadirachtin individually. The leaf discs dipped in acetone were used as negative control since acetone was used to dissolve the compound. In each plastic Petri dish $(1.5 \mathrm{~cm} \times 9$ $\mathrm{cm}$ ), a wet filter paper was placed to avoid early drying of the leaf discs. Single third instar larva of the respective insects was introduced into each Petri dish. Progressive consumption of treated and control leaves by the larvae after $24 \mathrm{~h}$ was assessed using the Leaf Area Meter (Delta-T Devices, Serial No. 15736 F 96, UK). Leaf area eaten by larva in the treatment was corrected from the negative control. Five replicates were maintained for each treatment with 10 larvae per replicate (total $\mathrm{n}=50$ ). The experiment was conducted at laboratory conditions $\left(27 \pm 2^{\circ} \mathrm{C}\right)$ with $14: 10 \mathrm{~h}$ photoperiod and $75 \pm 5 \%$ relative humidity. Antifeedant activity was calculated according to the formula of Bentley et al (1984).

\section{Larvicidal activity}

The protocol for larvicidal activity was as mentioned in antifeedant activity. After $24 \mathrm{~h}$ of treatment, the larvae were continuously maintained on the untreated fresh cotton and castor leaves for $H$. armigera and $S$. litura, respectively. Diet was changed every $24 \mathrm{~h}$. Larval mortality was recorded up to $96 \mathrm{~h}$ of treatment. Percent mortality was calculated according to Abbott's (1925) formula:

$\begin{aligned} & \text { Corrected } \\ & \text { mortality }\end{aligned}=\frac{\begin{array}{c}\% \text { mortality in treatment }-\% \\ \text { mortality in control }\end{array}}{100-\% \text { mortality in control }} \times \quad 100$ 


\section{Larval and pupal durations}

The treated larvae, which survived were continuously fed with fresh leaves. The larval duration was calculated after treated larvae became pupae. Pupal duration was calculated from the day of the emergence of adults from pupae.

\section{Adult emergence}

The treated pupae were maintained until the emergence of adults. Adult emergence was calculated by the number of emerging adults from the total number of pupae.

\section{Mosquito larvicidal activity}

Bioassays were studied using the method of World Health Organization (1996) with second and fourth instar larvae of An. stephensi and $C x$. quinquefasciatus. Flindersine was used at 2.5, 5, 10 and 20 ppm concentrations. Five replicate were maintained, each comprising 25 larvae (total $\mathrm{N}=$ 125). For the experiments, $249 \mathrm{~mL}$ dechlorinated tap water containing $0.1 \%$ Tween 80 and $1.0 \mathrm{~mL}$ of the required concentration of test solution were mixed.Tween80 was used as a negative control. Mortality rate was observed after $24 \mathrm{~h}$ of treatments.

\section{Statistical analysis}

All the data were analysed using one way ANOVA. Significant differences between the treatments were determined using DMRT tests $(\mathrm{P}$ $\leq 0.05$ ). Linear regression analysis was performed for all dose-response experimental data, and regression analysis was done between antifeedant and other biological activities. $\mathrm{LC}_{50}$ and $\mathrm{LC}_{90}$ values were calculated using probit analysis (Finney 1971).

\section{RESULTS}

\section{Antifeedant activity}

Table 1 shows the antifeedant activity of flindersine against $H$. armigera and $S$. litura. Maximum antifeedant activity of 84.24 and $78.07 \%$ was observed at $1000 \mathrm{ppm}$ concentrations against $H$. armigera and S. litura, respectively. All the tested concentrations showed more than $50 \%$ antifeedant activity against both the pests. Flindersine showed concentration dependent activity against both the pests. The regression $\left(\mathrm{R}^{2}\right)$ values of 0.91 and 0.87 were seen for $H$. armigera and $S$. litura. respectively. Flindersine and azadirachtin showed similar regression values $\left(\mathrm{R}^{2}\right)$ of 0.91 and 0.91 for $H$. armigera and $\mathrm{R}^{2} 0.88$ and 0.87 for $S$. litura, respectively. All the original data showed normality in Shapiro-Wilk test.

\section{Larvicidal activity}

Flindersine showed maximum larvicidal activity of 79.11 and $69.33 \%$ at $1000 \mathrm{ppm}$ concentration against $H$. armigera and $S$. litura, respectively. At $125 \mathrm{ppm}$ concentration, flindersine exhibited 24.88 and $22.44 \%$ larvicidal activity against $H$. armigera and $S$. litura, respectively. It exhibited good regression value $\left(\mathrm{R}^{2}\right)$ of 0.87 and 0.90 for $H$. armigera and $S$. litura, respectively. It exhibited $\mathrm{LC}_{50}$ values of 443.04 and $566.88 \mathrm{ppm}$ for larvicidal activity against $H$. armigera and $S$. litura, respectively (Table 2). Flindersine showed good regression value when compared to azadirachtin against both the pests. All the data did not show normality at Shapiro-Wilk test even after tansformation of data.

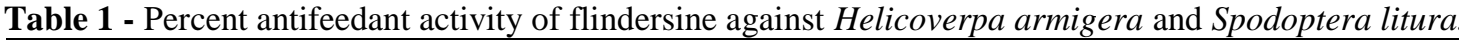

\begin{tabular}{|c|c|c|c|c|c|c|c|}
\hline Compound & $\begin{array}{c}\text { Concentration } \\
\text { ppm }\end{array}$ & H. armigera & $\mathbf{R}^{2}$ & $\begin{array}{c}\text { Regression } \\
\text { equation }\end{array}$ & S. litura & $\mathbf{R}^{2}$ & $\begin{array}{c}\text { Regression } \\
\text { equation }\end{array}$ \\
\hline \multirow{4}{*}{ Flindersine } & 125 & $53.64 \pm 3.38^{\mathrm{b}}$ & \multirow{4}{*}{0.91} & \multirow{4}{*}{$y=51.43 x+0.04$} & $50.66 \pm 3.28^{b}$ & \multirow{3}{*}{0.87} & \multirow{3}{*}{$y=49.99 x+0.03$} \\
\hline & 250 & $60.39 \pm 1.55^{\mathrm{c}}$ & & & $59.39 \pm 5.27^{\mathrm{c}}$ & & \\
\hline & 500 & $73.80 \pm 3.31^{\mathrm{d}}$ & & & $67.08 \pm 2.47^{\mathrm{d}}$ & & \\
\hline & 1000 & $84.24 \pm 1.56^{\mathrm{e}}$ & & & $78.07 \pm 4.57^{\mathrm{e}}$ & \multirow{5}{*}{0.88} & \multirow{6}{*}{$y=50.91 x+0.04$} \\
\hline \multirow{4}{*}{ Azadirachtin } & 125 & $54.54 \pm 3.67^{\mathrm{b}}$ & \multirow{4}{*}{0.91} & \multirow{4}{*}{$y=51.10 x+0.04$} & $51.76 \pm 3.25^{\mathrm{b}}$ & & \\
\hline & 250 & $62.43 \pm 3.38^{c}$ & & & $64.03 \pm 5.05^{\mathrm{cd}}$ & & \\
\hline & 500 & $74.13 \pm 2.82^{\mathrm{d}}$ & & & $73.89 \pm 5.21^{\mathrm{e}}$ & & \\
\hline & 1000 & $88.94 \pm 5.02^{\mathrm{f}}$ & & & $89.64 \pm 4.49^{\mathrm{f}}$ & & \\
\hline Control & & $3.02 \pm 1.39^{\mathrm{a}}$ & & & $2.09 \pm 1.12^{\mathrm{a}}$ & & \\
\hline
\end{tabular}

Means followed by the same letter do not differ significantly by using DMRT $(\mathrm{P} \leq 0.05)$ and complete regression equation. The results represent the mean \pm Standard Deviation. 
Table 2 - Percent larvicidal activity and effective concentration of flindersine against Helicoverpa armigera and Spodoptera litura.

\begin{tabular}{|c|c|c|c|c|c|c|c|c|c|}
\hline Compound & $\begin{array}{c}\text { Concentration } \\
\text { ppm }\end{array}$ & H. armigera & $\mathbf{L C}_{50}$ & $\mathbf{R}^{2}$ & $\begin{array}{l}\text { Regression } \\
\text { equation }\end{array}$ & S. litura & $\mathbf{L C}_{50}$ & $\mathbf{R}^{2}$ & $\begin{array}{c}\text { Regression } \\
\text { equation }\end{array}$ \\
\hline \multirow[t]{4}{*}{ Flindersine } & 125 & $24.88 \pm 4.75^{\mathrm{a}}$ & \multirow{4}{*}{443.04} & \multirow{4}{*}{0.87} & \multirow{4}{*}{$\begin{array}{l}y=24.17 x \\
+0.06\end{array}$} & $22.44 \pm 4.33^{\mathrm{a}}$ & \multirow{4}{*}{566.88} & \multirow{4}{*}{0.90} & \multirow{4}{*}{$\begin{array}{l}y=20.68 x \\
+0.05\end{array}$} \\
\hline & 250 & $39.55 \pm 3.97^{\mathrm{b}}$ & & & & $34.66 \pm 5.05^{\mathrm{b}}$ & & & \\
\hline & 500 & $62.44 \pm 5.79^{\mathrm{d}}$ & & & & $53.11 \pm 4.54^{\mathrm{d}}$ & & & \\
\hline & 1000 & $77.11 \pm 4.12^{\mathrm{e}}$ & & & & $69.33 \pm 1.49^{\mathrm{f}}$ & & & \\
\hline \multirow[t]{4}{*}{ Azadirachtin } & 125 & $48.88 \pm 4.64^{\mathrm{c}}$ & \multirow{4}{*}{145.69} & \multirow{4}{*}{0.72} & \multirow{4}{*}{$\begin{array}{l}y=52.14 x \\
+0.06\end{array}$} & $40.66 \pm 5.96^{\mathrm{c}}$ & \multirow{4}{*}{175.97} & \multirow{4}{*}{0.73} & \multirow{4}{*}{$\begin{array}{l}y=45.43 x \\
+0.06\end{array}$} \\
\hline & 250 & $66.00 \pm 3.65^{\mathrm{d}}$ & & & & $63.33 \pm 4.71^{\mathrm{e}}$ & & & \\
\hline & 500 & $97.77 \pm 4.96^{\mathrm{f}}$ & & & & $95.77 \pm 5.79^{\mathrm{g}}$ & & & \\
\hline & 1000 & $100.00 \pm 0.00^{\mathrm{f}}$ & & & & $100.00 \pm 0.00^{\mathrm{g}}$ & & & \\
\hline
\end{tabular}

Means followed by the same letter do not differ significantly by using DMRT $(\mathrm{P} \leq 0.05)$ and complete regression equations. The results represent the mean \pm Standard Deviation.

\section{Growth inhibitory activity}

Flindersine showed different kind of growth inhibitory activities against $H$. armigera and $S$. litura. It exhibited maximum larval duration of 14.66 and 15.10 days for $H$. armigera and $S$. litura, respectively at $1000 \mathrm{ppm}$ concentration. At $125 \mathrm{ppm}$ concentration, it also significantly increased the larval duration to 10.87 and 10.49 for $H$. armigera and $S$. litura, respectively, when compared to control (Table 3). Maximum pupal durations of 16.8 and 16.3 days were observed for H. armigera and S. litura, respectively at $500 \mathrm{ppm}$ concentration (Table 3). When compared to control, flindersine at all the concentrations showed significant activity against both the pests.
All the original larval-pupal duration data showed normality at Shapiro-Wilk test. No adult emergence was observed in $H$. armigera and $S$. litura at $1000 \mathrm{ppm}$ concentration of flindersine and at $125 \mathrm{ppm}$, it showed 68.45 and $63.80 \%$ adult emergence in $H$. armigera and $S$. litura respectively. At $500 \mathrm{ppm}$, less than $50 \%$ of adult emergence was observed in both the pests. Increased concentration of flindersine reduced the adult emergence. Statistically significant activity (adult emergence) was observed in all the concentrations of flindersine when compared to control. Due to post injective effect of flindersine, the larvae consumed low amount of untreated diet.

Table 3 - Growth inhibitory activity of flindersine against Helicoverpa armigera and Spodoptera litura.

\begin{tabular}{|c|c|c|c|c|c|c|c|}
\hline \multirow[b]{2}{*}{ Compound } & \multirow[b]{2}{*}{$\begin{array}{c}\text { Concentration } \\
\text { ppm }\end{array}$} & \multicolumn{3}{|c|}{ H. armigera } & \multicolumn{3}{|c|}{ S. litura } \\
\hline & & $\begin{array}{c}\text { Larval } \\
\text { duration days }\end{array}$ & $\begin{array}{c}\text { Pupal } \\
\text { duration days }\end{array}$ & $\begin{array}{c}\text { Adult } \\
\text { emergence }\end{array}$ & $\begin{array}{c}\text { Larval } \\
\text { duration days }\end{array}$ & $\begin{array}{c}\text { Pupal } \\
\text { duration days }\end{array}$ & $\begin{array}{c}\text { Adult } \\
\text { emergence }\end{array}$ \\
\hline \multirow{4}{*}{ Flindersine } & 125 & $10.87 \pm 0.57^{\mathrm{b}}$ & $12.17 \pm 0.32^{\mathrm{b}}$ & $68.45 \pm 4.83^{\mathrm{d}}$ & $10.49 \pm 0.22^{\mathrm{b}}$ & $11.9 \pm 0.28^{\mathrm{b}}$ & $63.80 \pm 6.38^{\mathrm{d}}$ \\
\hline & 250 & $12.14 \pm 0.54^{\mathrm{c}}$ & $14.33 \pm 0.52^{\mathrm{c}}$ & $50.57 \pm 9.34^{\mathrm{c}}$ & $11.79 \pm 0.38^{\mathrm{c}}$ & $13.9 \pm 0.65^{\mathrm{c}}$ & $46.66 \pm 10.27^{c}$ \\
\hline & 500 & $13.21 \pm 1.03^{\mathrm{de}}$ & $16.8 \pm 0.83^{\mathrm{d}}$ & $30.00 \pm 4.56^{\mathrm{b}}$ & $13.01 \pm 0.21^{\mathrm{d}}$ & $16.3 \pm 0.67^{\mathrm{d}}$ & $36.00 \pm 3.65^{\mathrm{b}}$ \\
\hline & 1000 & $14.66 \pm 0.77^{\mathrm{f}}$ & - & $00.00 \pm 0.00^{\mathrm{a}}$ & $15.10 \pm 0.41^{\mathrm{f}}$ & - & $00.00 \pm 0.00_{\mathrm{a}}$ \\
\hline \multirow{4}{*}{ Azadirachtin } & 125 & $12.61 \pm 0.97^{\mathrm{cd}}$ & $16.6 \pm 0.89^{d}$ & $30.00 \pm 9.35^{\mathrm{b}}$ & $12.03 \pm 0.47^{\mathrm{c}}$ & $15.8 \pm 0.27^{\mathrm{d}}$ & $42.66 \pm 7.22^{\mathrm{bc}}$ \\
\hline & 250 & $13.73 \pm 0.56^{\mathrm{ef}}$ & - & $00.00 \pm 0.00^{\mathrm{a}}$ & $14.23 \pm 0.22^{\mathrm{e}}$ & - & $00.00 \pm 0.00^{\mathrm{a}}$ \\
\hline & 500 & - & - & - & - & - & - \\
\hline & 1000 & - & - & - & - & - & - \\
\hline Control & & $9.21 \pm 0.41^{\mathrm{a}}$ & $9.92 \pm 0.26^{\mathrm{a}}$ & $95.55 \pm 6.08^{\mathrm{e}}$ & $9.35 \pm 0.19^{\mathrm{a}}$ & $10.11 \pm 0.35^{\mathrm{a}}$ & $97.77 \pm 4.96^{\mathrm{e}}$ \\
\hline
\end{tabular}

Means followed by the same letter do not differ significantly by using DMRT $(\mathrm{P} \leq 0.05)$. Values were Mean $\pm \mathrm{SD}$.

\section{Regression value}

The regression value is an important factor. All the biological activities are directly dependent on the consumption of food. After $24 \mathrm{~h}$ of treatment, the larvae were unable to feed normally. The larvalpupal durations and adult emergence varied when compared to control due to post injective effects of flindersine. It showed good regression values between antifeedant and larvicidal activities $\left(\mathrm{R}^{2}\right.$ 0.94 and 0.87), antifeedant and larval duration $\left(\mathrm{R}^{2}\right.$ $0.79 \& 0.89)$, antifeedant and pupal duration $\left(\mathrm{R}^{2}\right.$ 0.91 and 0.79$)$ and antifeedant and adult emergence $\left(\mathrm{R}^{2} 0.93 \& 0.88\right)$ for $H$. armigera and $S$. litura, respectively (Table 4). 


\section{Mosquito larvicidal activity}

In the present study, flindersine exhibited $100 \%$ larvicidal activity against An. stephensi and $C x$. quinquefasciatus at $20 \mathrm{ppm}$ concentration (Table 5). It manifested least $\mathrm{LC}_{50}$ value of $1.68 \mathrm{ppm}$ (95\% confidential interval 1.11-2.14 ppm) against $2^{\text {nd }}$ instar larvae of An. Stephensi, followed by 2.71 ppm (95\% confidential interval 2.18-3.15 ppm) for $4^{\text {th }}$ instar larvae of An. stephensi. In the case of $C x$. quinquefasciatus, flindersine exhibited $\mathrm{LC}_{50}$ value of 2.90 (95\% confidential interval $2.38-3.35 \mathrm{ppm}$ ) and 4.19 (95\% confidential interval 3.74-4.61 ppm) against $2^{\text {nd }}$ and $4^{\text {th }}$ instar larvae, respectively. All the tested concentrations exhibited more than $40 \%$ larvicidal activity against both vector mosquitoes. Toxicity was concentration dependent. At $10 \mathrm{ppm}$, flindersine exhibited more larvicidal activity against both the tested mosquitoes.

Table 4 - Linear regression relationship between biological activities on treated insects.

\begin{tabular}{|c|c|c|c|c|c|c|c|}
\hline Regression between & Insects & Treatment & $\mathbf{R}$ & $\mathbf{R}^{2}$ & $\begin{array}{c}\text { Regression } \\
\text { equation }\end{array}$ & $\mathbf{F}$ & $\mathbf{P}$ \\
\hline \multirow{4}{*}{$\begin{array}{l}\text { Antifeedant and } \\
\text { larvicidal }\end{array}$} & \multirow{2}{*}{ H. armigera } & Flindersin & 0.97 & 0.94 & $y=38.33 x+0.58$ & 295.9 & 0.00 \\
\hline & & Azadirachtin & 0.87 & 0.76 & $y=28.30 x+0.53$ & 56.4 & 0.00 \\
\hline & \multirow{2}{*}{ S. litura } & Flindersin & 0.93 & 0.87 & $y=39.38 x+0.54$ & 122.9 & 0.00 \\
\hline & & Azadirachtin & 0.89 & 0.78 & $y=31.06 x+0.52$ & 64.8 & 0.00 \\
\hline \multirow{4}{*}{$\begin{array}{l}\text { Antifeedant and larval } \\
\text { duration }\end{array}$} & \multirow{2}{*}{ H. armigera } & Flindersin & 0.89 & 0.79 & $y=-21.44 x+7.01$ & 65.8 & 0.00 \\
\hline & & Azadirachtin & 0.43 & 0.19 & $y=26.68 x+2.42$ & 1.87 & 0.21 \\
\hline & \multirow[b]{2}{*}{ S. litura } & Flindersin & 0.94 & 0.89 & $y=-8.9 x+5.8$ & 129.4 & 0.00 \\
\hline & & Azadirachtin & 0.80 & 0.63 & $y=-7.8 x+4.9$ & 13.73 & 0.00 \\
\hline \multirow{4}{*}{$\begin{array}{l}\text { Antifeedant and pupal } \\
\text { duration }\end{array}$} & \multirow{2}{*}{ H. armigera } & Flindersin & 0.95 & 0.91 & $y=-0.18 x+4.3$ & 126.3 & 0.00 \\
\hline & & Azadirachtin & 0.76 & 0.57 & $y=106.2 x+-3.1$ & 4.01 & 0.14 \\
\hline & \multirow{2}{*}{ S. litura } & Flindersin & 0.90 & 0.79 & $y=10.37 x+3.5$ & 49.13 & 0.00 \\
\hline & & Azadirachtin & 0.41 & 0.21 & $y=-68.01 x+7.6$ & 2.01 & 0.25 \\
\hline \multirow{4}{*}{$\begin{array}{l}\text { Antifeedant and Adult } \\
\text { emergence }\end{array}$} & \multirow{2}{*}{ H. armigera } & Flindersin & 0.94 & 0.93 & $y=84.82 x+-0.46$ & 259.8 & 0.00 \\
\hline & & Azadirachtin & 0.73 & 0.53 & $y=61.92 x+-0.23$ & 9.1 & 0.02 \\
\hline & \multirow{2}{*}{ S. litura } & Flindersin & 0.94 & 0.88 & $y=78.89 x+-0.41$ & 129.3 & 0.00 \\
\hline & & Azadirachtin & 0.87 & 0.74 & $y=64.01 x+-0.29$ & 23.88 & 0.00 \\
\hline
\end{tabular}

* significant at $(\mathrm{P} \leq 0.05)$ level. Values were Mean $\pm \mathrm{SD}$.

Table 5 - Larvicidal activity of Flindersine against vector mosquitoes of Anopheles stephensi and Culex quinquefasciatus.

\begin{tabular}{|c|c|c|c|c|c|c|c|c|c|}
\hline \multirow[t]{2}{*}{ Instars } & \multirow[t]{2}{*}{$\begin{array}{c}\text { Concentration } \\
(\text { ppm })\end{array}$} & \multirow[t]{2}{*}{$\begin{array}{c}\text { \% larvicidal } \\
\text { activity }\end{array}$} & \multirow[t]{2}{*}{$\mathbf{L C}_{50}$} & \multicolumn{2}{|c|}{$\begin{array}{c}95 \% \text { confidential } \\
\text { interval }\end{array}$} & \multirow[t]{2}{*}{$\mathbf{L C}_{99}$} & \multicolumn{2}{|c|}{$\begin{array}{c}95 \% \text { confidential } \\
\text { interval }\end{array}$} & \multirow[t]{2}{*}{$\chi^{2}$} \\
\hline & & & & Lower & Upper & & Lower & Upper & \\
\hline & & & & \multicolumn{5}{|c|}{ Cx. quinquefasciatus } & \\
\hline \multirow[t]{4}{*}{$2^{\text {nd }}$} & 2.5 & $48.73 \pm 3.57$ & & & & & & & \\
\hline & 5 & $62.74 \pm 4.51$ & & & & & & & \\
\hline & 10 & $90.19 \pm 4.40$ & 2.90 & 2.38 & 3.35 & 15.76 & 14.52 & 17.34 & 16.20 \\
\hline & 20 & $100.0 \pm 0.00$ & & & & & & & \\
\hline \multirow[t]{5}{*}{$4^{\text {th }}$} & 2.5 & $40.45 \pm 3.68$ & & & & & & & \\
\hline & 5 & $54.53 \pm 4.58$ & & & & & & & \\
\hline & 10 & $81.77 \pm 3.81$ & 4.19 & 3.74 & 4.61 & 18.21 & 16.89 & 19.54 & 13.41 \\
\hline & 20 & $100.0 \pm 0.00$ & & & & & & & \\
\hline & & \multicolumn{8}{|c|}{ An. stephensi } \\
\hline \multirow[t]{4}{*}{$2^{\text {nd }}$} & 2.5 & $58.34 \pm 2.11$ & & & & & & & \\
\hline & 5 & $76.63 \pm 4.70$ & 168 & 11 & 214 & 1176 & 1070 & 1304 & 2615 \\
\hline & 10 & $97.53 \pm 3.61$ & 1.68 & 1.11 & 2.14 & 11.76 & 10.79 & 13.04 & 26.15 \\
\hline & 20 & $100.0 \pm 0.00$ & & & & & & & \\
\hline \multirow[t]{4}{*}{$4^{\text {th }}$} & 2.5 & $47.42 \pm 3.14$ & & & & & & & \\
\hline & 5 & $68.39 \pm 4.06$ & & & & & & & \\
\hline & 10 & $90.85 \pm 3.47$ & 2.71 & 2.18 & 3.15 & 15.04 & 13.86 & 16.55 & 12.21 \\
\hline & 20 & $100.0 \pm 0.00$ & & & & & & & \\
\hline
\end{tabular}

Values were Mean \pm SD. 


\section{DISCUSSION}

\section{Antifeedant activity}

Flindersine isolated from $T$. asiatica was studied for biological activities against $H$. armigera and $S$. litura. Generally, alkaloid compounds act as a stomach poison. In the present study, flindersine showed maximum antifeedant activity of 84 and $78 \%$ against $H$. armigera and $S$. litura, respectively at $1000 \mathrm{ppm}$ concentration, which confirmed the findings of Cornelius et al. (2009) who reported that erythrina alkaloids of $(+)-11 \beta-$ methoxy-10-oxoerysotramidine from Erythrina latissima (Fabaceae) showed $80.9 \%$ antifeedant activity against $S$. littoralis Boisduval at 1000 ppm concentration. Furoquinoline alkaloids (dictamnine and evolitrine) derived from Evodia lunuankenda (Rutaceae) showed antifeedant activity against $S$. litura (Jagadeesh et al. 2000). The present result was also supported by the findings of Il-Kwon et al (2000) who reported that isoquinoline alkaloids of palmatine iodide and berberine chloride from Coptis japonica (Ranunculaceae) showed more than $75 \%$ antifeedant activity against Hyphantria cunea Drury. Dihydro- $\beta$-agarofuran, derived from Parnassia wightiana (Celastraceae) showed high antifeedant activity against Mythimna separata Walker (Jiang-Jiang et al. 2013).

\section{Larvicidal activity}

In this study, flindersine showed $\mathrm{LC}_{50}$ values of 443.04 and 566.88 ppm against $H$. armigera and $S$. litura, respectively. Similarly, Emam et al. (2009) reported that furocoumarin alkaloid- 3(2",2"dimethyl butenyl) 3' - hydroxy dihydrofuropsoralen and quinolone alkaloidrutamine exhibited larvicidal activity against $S$. littoralis with $\mathrm{LC}_{50}$ values of 1598 and 1215 ppm, respectively. Pipernonaline and piperoctadecalidine isolated from Piper longum (Piperaceae) showed insecticidal activity with $\mathrm{LC}_{50}$ values of 530 and $235 \mathrm{ppm}$, respectively against Myzus persicae Sulzer and also showed insecticidal activity against Nilaparvata lugens (Stål) and S. litura (Park et al. 2002). Moreira et al. (2007) reported that 5,6,7,8,3',4', 5'heptamethoxyflavone and 5,6,7,8,3'pentamethoxy-4', $5^{\prime}$-methylenedioxyflavone from Ageratum conyzoides (Asteraceae) showed insecticidal activity against Rhyzopertha dominica Fabricius, Diaphania hyalinata Linnaeus, Musca domestica Linnaeus and Periplaneta americana Linnaeus.

\section{Growth inhibition}

Flindersine showed growth inhibitory activities such as increased larval-pupal duration and reduction in adult emergence in $H$. armigera and $S$. litura. The present finding corroborated with those of Acheuk and Doumandji-Mitiche (2013) who reported that the extracts containing alkaloid from Pergularia tomentosa (Apocynaceae) affected the larval duration and adult emergence of Locusta migratoria cinerascens Fabricius; the larval duration increased up to 18.0 days after the treatments when compared to control and adult emergence significantly decreased. Hexane extracts and their fractions containing alkaloids from Atalantia monophylla (Rutaceae) completely prevented the adult emergence of $H$. armigera (Baskar et al. 2009). In the present study, larvalpupal duration increased and adult emergence was reduced. These findings were in agreement with Sakr et al. (2013) who reported that 5-hydroxy7,4'-dimethoxy-flavon-3-ol and 5-hydroxy-7methoxy-2-(4'-methoxy-phenyl)-chromen-4-one isolated from a dichloromethane extract of Hyptis brevipes (Lamiaceae) showed growth inhibitory activity against $S$. littoralis. Similarly, many workers have reported that naturally purified, semi purified compounds and extracts exhibited growth inhibitory activity against agricultural pests. Baskar and Ignacimuthu (2012) reported that ononitol monohydrate from Cassia tora (Fabaceae) increased the larval-pupal durations and inhibited the adult emergence of $H$. armigera and $S$. litura. Hexane and ethyl acetate extracts from the leaf and root of Aristalochia tagala (Aristolochiaceae) increased the larval-pupal duration and reduced the adult emergence (Baskar et al. 2011). In the present study, flindersine exhibited no adult emergence at higher concentration in $H$. armigera and $S$. litura. These findings supported the earlier findings of Munoz et al. (2013) who reported that hexane, ethyl acetate and methanol extracts from $C$. talcana reduced the adult emergence of $S$. frugiperda.

\section{Regression value}

Flindersine showed high regression value between antifeedant and other parameters in this study. Flindersine showed good regression value (R 0.94) between antifeedant and adult emergence for the two tested insects. These finding corroborated with 
Gonzalez-Coloma et al. (1995) reported that $11 \beta$ acetoxy-5-angeloyloxy-silphinen-3-one from Senecio palmensis (Asteraceae) showed good regression value ( $R$ 0.82) between relative consumption and relative growth rates in Leptinotarsa decemlineata Say. Flindersine showed good regression value between the concentration and antifeedant activity of the tested insects. Similarly, Wheeler and Isman (2001) reported that Trichilia americana (Meliaceae) extracts showed high regression value $\left(\mathrm{R}^{2} 0.96\right)$ between relative consumption and relative growth rate in S. litura. Osthol [2H-1-Benzopyran-2-one, 7-methoxy-8-(3-methyl-2-butenyl) from chloroform root extract of Clausena anisata (Rutaceae) showed high regression $\left(\mathrm{R}^{2}\right.$ 0.82) between concentration and antifeedant activity in $H$. armigera (Pitan et al. 2009). In this study, flindersine showed more than $0.80 \mathrm{R}$ value between antifeedant and larvicidal activity in $H$. armigera and $S$. litura. These findings corroborated with the findings of Baskar et al. (2014) who reported that friedelin showed more than $0.80 \mathrm{R}$ value between antifeedant and larvicidal activity against $H$. armigera and $S$. litura.

\section{Mosquito larvicidal activity}

In the present study, flindersine exhibited $100 \%$ larvicidal activity with less than $5 \mathrm{ppm} \mathrm{LC}_{50}$ against An. stephensi and $C x$. quinquefasciatus. This result corroborated with the findings of Maheswaran and Ignacimuthu (2013) who reported that confertifolin from Polygonum hydropiper (Polygonaceae) exhibited less than 5 ppm $\mathrm{LC}_{50}$ against An. stephensi and $C x$. quinquefasciatus. Pectolinaringenin from Clerodendrum phlomidis (Lamiaceae) exhibited larvicidal activity against $C x$. quinquefasciatus and A. aegypti (Muthu et al. 2012). Similarly, essential oil and their compounds sabinene, biofloratriene, borneol and $\beta$-Bisabolol from Clausena dentate (Rutaceae) exhibited larvicidal activity against $A$. aegypti (Rajkumar and Jebanesan 2010). Lima et al. (2014) reported that Mentha x villosa (Lamiaceae) essential oil showed larvicidal activity against $A$. aegypti. Root extract of Tragia involucrate (Euphorbiaceae) exhibited more than 90\% larvicidal activity against $C \boldsymbol{x}$. quinquefasciatus (Bhattacharya and Chandra 2014).

\section{CONCLUSION}

In the present study flindersine, an alkaloid showed significant antifeedant, larvicidal, growth inhibitory activities against $H$. armigera and $S$. litrua. It showed $100 \%$ larvicidal activity against vector mosquitoes at $20 \mathrm{ppm}$ concentration. Larvicidal is main methods to control and manage the vector mosquitoes. Antifeedancy is a primary factor, which influences larval death, larval-pupal duration and adult emergence of the pests. High correlation between antifeedant and larvicidal and growth inhibitory values were recorded. Flindersine could be a suitable compound to develop a new botanical pesticide formulation to control vector mosquitoes and agricultural pests.

\section{ACKNOWLEDGMENT}

This project was supported by King Saud University, Deanship of Scientific Research, Addiriyah Chair for Environmental Studies

\section{REFERENCES}

Abbott WS. A method of computing the effectiveness of an insecticide. J Econ Entomol. 1925; 18: 265-267.

Acheuk F, Doumandji-Mitiche B. Insecticidal activity of alkaloids extract of Pergularia tomentosa (Asclepiadaceae) against Locusta migratoria cinerascens (Fabricius 1781) (Orthoptera:Acrididae). Int J Sci Adv Technol. 2013; 3: 8-13.

Akhtar Y, Isman MB, Niehaus LA, Lee CH, Lee HS. Antifeedant and toxic effects of naturally occurring and synthetic quinines to the cabbage looper, Trichoplusia ni. Crop Protect. 2012; 31: 8-14.

Ayyanar M, Ignacimuthu S. Traditional knowledge of Kani tribals in Kouthalai of Tirunelveli hills, Tamil Nadu, India. J. Ethnopharmacol. 2005; 102: 246-255.

Balick MJ, Elisabetsky E, Laird SA. Medicinal Resources of the Tropical Forest: Biodiversity and its Importanceto Human Health. Columbia University Press, New York, 1995, 440 pp.

Baskar K, Duraipandiyan V, Ignacimuthu S. Bioefficacy of friedelin (Triterpenoid) against Helicoverpa armigera (Hub.) and Spodoptera litura (Fab.) (Lepidoptera: Noctuidae). Pest Manage Sci. 2014; 70: 1877-1883.

Baskar K, Ignacimuthu S. Antifeedant, larvicidal and growth inhibitory effect of ononitol monohydrate isolated from Cassia tora L. against Helicoverpa armigera (Hub.) and Spodoptera litura (Fab.) (Lepidoptera: Noctuidae). Chemosphere. 2012; 88: 384-388 
Baskar K, Kingsley S, Vendan SE, Paulraj MG, Duraipandiyan V, Ignacimuthu S. Antifeedant, larvicidal and pupicidal activities of Atalantia monophylla (L) Correa against Helicoverpa armigera Hubner (Lepidoptera: Noctuidae). Chemosphere. 2009; 75: 355-359.

Baskar K, Sasikumar S, Muthu C, Kingsley S, Ignacimuthu S. Bioefficacy of Aristolochia tagala against Spodoptera litura Fab. (Lepidoptera:Noctuidae). Saudi J Biol Sci. 2011; 18: 23-27.

Bentley MD, Leonard DE, Stoddard WF, Zalkow LH. Pyrrolizidine alkaloids as larval feeding deterrents for spruce budworm, Choristoneura fumiferana (Lepidoptera: Tortricidae). Ann Entomol Soc Am. 1984; 77: 393-397.

Bhattacharya K, Chandra G. Phagodeterrence, larvicidal and oviposition deterrence activity of Tragia involucrata L. (Euphorbiaceae) root extractives against vector of lymphatic filariasis Culex quinquefasciatus (Diptera: Culicidae). Asian Pac J Trop Dis. 2014; 4: S226-S232.

Borah R, Kalita MC, Kar A, Talukdar AK. Larvicidal efficacy of Toddalia asiatica (Linn.) Lam against two mosquito vectors Aedes aegypti and Culex quinquefasciatus. African J Biotechnol. 2010; 9: 2527-2530.

Cornelius WW, Akenga T, Obiero GO, Lutta KP. Antifeedant activities of the erythrinaline alkaloids from Erythrina latissima against Spodoptera littoralis (Lepidoptera: Noctuidae). Rec Nat Prod. 2009; 3: 96103.

Dhir BC, Mohapatra HK, Senapati B. Assessment of crop loss in groundnut due to tobacco caterpillar Spodoptera litura (F). Ind J Plant Protect. 1992; 20: 215-217.

Duraipandiyan V, Ignacimuthu S. Antibacterial and antifungal activity of flindersine isolated from the traditional medicinal plant, Toddalia asiatica (L.) Lam. J Ethnopharmacol. 2009; 123: 494-498.

Emam AM, Swelam ES, Megally NY. Furocoumarin and quinolone alkaloid with larvicidal and antifeedant activities isolated from Ruta chalepensis Leave. J Nat Products. 2009; 2: 10-22.

Finney DJ. Probit analysis. 3rd ed. Cambridge University Press. London. 1971.

Gonzalez-Coloma A, Reina M, Cabrera R, Castannera P, Gutierrez C. Antifeedant and toxic effects of sesquiterpenes from Senecio palmensis to colorado potato beetle. J Chem Ecol. 1995; 21: 1255-1270.

Il-Kwon P, Hoi-Seon L, Sang-Gil L, Ji-Doo P, YoungJoon A. Antifeeding activity of isoquinoline alkaloids identified in Coptis japonica roots against Hyphantria cunea (Lepidoptera: Arctiidae) and Agelastica coerulea (Coleoptera: Galerucinae). J Econ Entomol. 2000; 93: 331-335.
Isman MB. Insect antifeedants. Pestic Outlook. 2002; 13: $129-176$.

Jagadeesh SG, Krupadanam GLD, Srimannarayana G. Antifeedant activity of the constituents of Evodia lunu-ankenda. Ind J Chem. 2000; 39B: 475-476.

Jiang-Jiang T, Fei-Yu Z, Dong-Mei W, Jun-Mian T, Shuai D, Jin-Ming G. Semisynthesis and antifeedant activity of new derivatives of a dihydro- $\beta$-agarofuran from Parnassia wightiana. Int J Molec Sci. 2013; 14: 19484-19493.

Koul O, Shankar JS, Mehta N, Taneja SC, Tripathi AK, Dhar KL. Bioefficacy of crude extracts of Aglaia species (Meliaceae) and some active fractions against lepidopteran larvae. J Appl Entomol. 1997; 121: 245248.

Kriti JS, Dar MA, Khan ZH. Biological and taxonomic study of agriculturally important noctuid pests of Kashmir. World Journal of Agricultural Research. 2014; 2:82-87.

Kumar BVN, Reghupathy A. Status of insecticide resistance in tobacco caterpillar, Spodoptera litura (Fab.) in Tamil Nadu. Pestic Res J. 2001; 13: 86-89.

Lima TC, da Silva TKM, Silva FL, Barbosa-Filho JM, Marques MOM, Santos RLC, Cavalcanti SCDH, de Sousa DP. Larvicidal activity of Mentha x villosa Hudson essential oil, rotundifolone and derivatives. Chemosphere. 2014; 104: 37-43.

Liu XC, Dong HW, Zhou L, Du SS, Liu ZL. Essential oil composition and larvicidal activity of Toddalia asiatica root against the mosquito Aedes albopictus (Diptera:Culicidae). Parasitol Res. 2013; 112: 1197 1203.

Maheswaran R, Ignacimuthu S. A novel herbal formulation against dengue vector mosquitoes Aedes aegypti and Aedes albopictus. Parasitol Res. 2012; 110: 1801-1813.

Maheswaran R, Ignacimuthu S. Bioefficacy of essential oil from Polygonum hydropiper L. against mosquitoes, Anopheles stephensi and Culex quinquefasciatus. Ecotoxicol Environ Safety. 2013; 97: 26-31.

Manjunath TM, Bhatnagar VS, Pawar CS, Sithanantham S. Economic importance of Heliothis spp. in India and an assessment of their natural enemies and host plants. In: Proceedings of the Workshop on Biological Control of Heliothis: increasing the effectiveness of natural enemies. New Delhi, India. 1989; 197-228 pp.

Moreira MD, Picanco MC, Barbosa LCA, Guedes RNC, Barros EC, Campos MR. Compounds from Ageratum conyzoides: isolation, structural elucidation and insecticidal activity. Pest Manag Sci. 2007; 63: 615-621.

Mullai K, Jebanesan A, Pushpanathan T. Effect of bioactive fractions of Citrullus vulgaris Schrad. leaf extract against Anopheles stephensi and Aedes aegypti. Parasitol Res. 2008; 102: 951-955. 
Munoz E, Lamilla C, Marin JC, Alarcon J, Cespedes CL. Antifeedant, insect growth regulatory and insecticidal effects of Calceolaria talcana (Calceolariaceae) on Drosophila melanogaster and Spodoptera frugiperda. Ind Crops Prod. 2013; 42: 137-144.

Muthu C, Reegan AD, Kingsley S, Ignacimuthu S. Larvicidal activity of pectolinaringenin from Clerodendrum phlomidis L. against Culex quinquefasciatus Say and Aedes aegypti L. (Diptera: Culicidae). Parasitol Res. 2012; 111: 1059-1065.

Nyahanga T, Jondiko JI, Manguro LA, Orwa JA. Antiplasmodial and larvicidal compounds of Toddalia asiatica root and bark. J Chem Sci. 2013; 125: 1115-1121.

Park BS, Lee SE, Choi WS, Jeong CY, Song C, Cho KY. Insecticidal and acaricidal activity of pipernonaline and piperoctadecalidine derived from dried fruits of Piper longum L. Crop Protect. 2002; 21: 249-251.

Pitan OOR, Ayelaagbe OO, Wang HL, Wang CZ. Identification, isolation and characterization of the antifeedant constituent of Clausena anisata against Helicoverpa armigera (Lepidoptera: Noctuidae). Insect Sci. 2009; 16: 247-253.

Rajasekariah GR, Parab PB, Chandrashekar R, Deshpande L, Subrahmanyan D. Pattern of Wuchereria bancrofti microfilaraemia in young and adolescent school children in Bessein, India, an endemic area for lymphatic filariasis. Ann Trop Med Parasitol. 1991; 85: 663-665.

Rajkumar S, Jebanesan A. Chemical composition and larvicidal activity of leaf essential oil from Clausena dentata (Willd) M. Roam. (Rutaceae) against the chikungunya vector, Aedes aegypti Linn. (Diptera: Culicidae). J Asia-Pacific Entomol. 2010; 13: 107109.

Ramana VV, Reddy GPV, Krishnamurthy MM. Synthetic pyrethroids and other bait formulation in the control of Spodoptera litura (Fab.) attacking rabi groundnut. J Pesticides. 1988; 1: 522-524.

Ramasubramaniam T, Regupathy A. Pattern of cross resistance in pyrethroid selected populations of Helicoverpa armigera from India. J Appl Ent. 2004; 128, 583-587.

Ramya S, Alaguchamy N, Mohan PJJ, Kalayanasundaram M, Jayakumararaj R. Antifeedant efficacy of Helicoverpa armigera on leaf extracts of selected medicinal plants. J Ecobiol. 2009; 25: 263270.

Sakr HH, Roshdy SH, El-Seedi HR. Hyptis brevipes (Lamiaceae) extracts strongly inhibit the growth and development of Spodoptera littoralis (Boisd.) larvae (Lepidoptera: Noctuidae). J Appl Pharmaceutical Sci. 2013; 3: 83-88.
Salehzadeh A, Jabbar A, Jennens L, Ley SV, Annadurai RS, Adams R, Strang RHC. The effect of phytochemical pesticides on the growth of cultured invertebrate and vertebrate cells. Pest Manage Sci. 2002; 58: 268-276.

Senthilkumar A, Kannathasan K, Venkatesalu V. Chemical constituents and larvicidal property of the essential oil of Blumea mollis (D. Don) Merr. against Culex quinquefasciatus. Parasitol Res. 2008; 103: 959-962.

Sundararajan G, Kumuthakalavalli R. Antifeedant activity of aqueous extract of Gnidia glauca Gilg. and Toddalia asiatica Lam. on the gram pod borer, Helicoverpa armigera (Hbn). J Environ Biol. 2001; 22: 11-14.

Talekar NS, Opena RT, Hanson P. Helicoverpa armigera management: a review of AVRDC's research on host plant resistance in tomato. Crop Protect. 2006; 5: 461-467.

Talukder FA, Howse PE. Laboratory evaluation of toxic and repellent properties of the pithraj tree, Aphanamixis polystachya Wall and Parker, against Sitophilus oryzae (L.). Int J Pest Manage. 1994; 40: 274-279.

Tavares WDS, Freitas SDS, Grazziotti GH, Parente LML, Liao LM, Zanuncio JC. Ar-turmerone from Curcuma longa (Zingiberaceae) rhizomes and effects on Sitophilus zeamais (Coleoptera: Curculionidae) and Spodoptera frugiperda (Lepidoptera: Noctuidae). Ind Crops Prod. 2013; 46: 158-164.

Usher BF, Bernays EF, Berbehenn RV. Antifeedant tests with larvae of Pseudaletia unipuncta: variability of behavioral response. Entomol Exp Appl. 1988; 48: 203-212.

Wheeler DA, Isman MB. Antifeedant and toxic activity of Trichilia americana extract against the larvae of Spodoptera litura. Entomol Exp Appl. 2001; 98: 9-16.

World Health Organization. Report of the WHO informal consultation on the evaluation and testing insecticides.CTD/WHO PES/IC/96.1. 1996; pp 69

Youn YN, Seo MJ, Shin JG, Jang C, Yu YM. Toxicity of greenhouse pesticides to multicolored Asian lady beetles, Harmonia axyridis (Coleoptera:Coccinellidae). Biol Control. 2003; 28: 164-170.

Zhong G, Liu J, Weng Q, Hu M, Luo J. Laboratory and field evaluations of rhodojaponin-III against the imported cabbage worm Pieris rapae (L.) (Lepidoptera: Pieridae). Pest Manag Sci. 2006; 62: 976-981. 